EESTI NSV TEADUSTE AKADEEMIA TOIMETISED. XII KÖIDE

FOOSIKA-MATEMAATIKA- JA TEHNIKATEADUSTE SEERIA. 1963, NR. 2

ИЗВЕСТИЯ АҚАДЕМИИ НАУК ЭСТОНСКОЙ ССР. ТОМ ХІІ СЕРИЯ ФИЗИКО-МАТЕМАТИЧЕСКИХ И ТЕХНИЧЕСКИХ НАУК. 1963, № 2

\title{
АХРОМАТИЗМ ПРОСВЕТЛЕНИЯ ИЛИ АНТИПРОСВЕТЛЕНИЯ ПОГЛОЩАЮЩИХ ПЛЕНОК
}

\author{
П. КАРД, \\ член-корреспондент Академии наук Эстонской ССР
}

На основе общей формулы меры просветления поглощающей пленки дан анализ условий ахроматичности просветления или антипросветления. Показано, что ахроматичность просветления достижима только в сравнительно узком интервале спектра, тогда как антипросветление может быть сделано слабо зависящим от длины волны в широкой спектральной области.

\section{Введение}

Теория просветления поглошающих пленок развита автором в нескольких статьях $\left[{ }^{1-4}\right]$, однако вопрос об ахроматичности просветления в них не рассматривался. Цель настоящей статьи состоит в заполнении этого пробела, т. е. в обсуждении условий, необходимых для осуществления просветления в более или менее широком спектральном интервале. При этом оказывается целесообразным рассматривать вместе с просветлением также и антипросветление. Напомним (см. [1]), что просветлением по. глощаюшей пленки называется уменьшение отношения $A / D$, где $A$ - коэффициент поглощения, $D$ - коэффициент пропускания. Аналогично, антипросветление означает увеличение $A / D$.

Изменение значения отношения $A 7 D$ в ту или другую сторону может быть достигнуто путем помещения между данной поглощающей (в общем случае многослойной) пленкой и подложкой должным образом подобранной непоглощающей пленки. Это так называемое внешнее просветление (или антипросветление). В этом случае (анти)просветляемая пленка считается полностью заданной и желаемый результат достигается с помощью добавочной пленки, внешней по отношению к данной.

Целесообразно, однако, кроме внешнего, учитывать также внутреннее (анти)просветление. Под этим термином подразумевается следующее. Если данная пленка состоит из нескольких поглощающих слоев, между которыми находятся непоглощающие прослойки, то можно поставить вопрос о таком выборе этих прослоек, который (совместно с внешним (анти)просветлением) привел бы к возможно малому (или возможно большому) значению $A / D$. Таким образом, в этой постановке вопроса заданными считаются только поглощающие слои, образующие так называемый остов поглощающей пленки (см. $\left.{ }^{2}\right]$ ). Слои же, находящиеся между слоями остова, а также между последним слоем остова и подложкой, должны быть подобраны так, чтобы в. целом получилась пленка с возможно малым или, наоборот, с возможно большим значением $A / D$. Надлежащий подбор слоев между слоями остова и называется внутренним (анти)просветлением. Ясно, что с практической точки зрения учет внутреннего (анти)просветления наряду с внешним является естественным и целесообразным. 
В самом деле, если мы хотим получить пленку, обладающую возможно малым поглощением, но содержащую, например, два металлических слоя. то, очевидно, следует, кроме внешнего просветления, позаботиться также и о таком заполнении промежутка между этими слоями, которое тоже служило бы поставленной цели.

\section{1. Условия внешнего просветления или антипросветления}

Рассмотрим сначала внешнее (анти)просветление. Оно состоит в добавлении к данной пленке некоторой добавочной пленки. Выделив в качестве промежуточного ее первый слой, прилегающий к данной пленке, обозначим остальную ее часть индексом 2 , а данную пленку индексом 1. Величины, относящиеся ко всей комбинированной пленке, будем писать без индекса. Примем для простоты показатели преломления промежуточного слоя и подложки равными. Можно показать, что общность наших выводов этим существенно не нарушается.

Назовем мерой просветления величину

$$
s=1-\frac{A / D}{A_{1} / D_{1}}
$$

Это название оправдано тем, что в случае просветления $0<s<1$, причем, чем больше $s$, тем значительнее просветление. При $s<0$, наоборот, имеет место антипросветление, причем $1-s$ означает, во сколько раз увеличилось отношение $A / D$. В дальнейшем под просветлением будет подразумеваться как положительное просветление (т. е. просветление в собственном смысле), так и отрицательное просветление (т. е. антипросветление).

Для меры просветления можно легко вывести формулу (см. $\left.\left[{ }^{1,2}\right]\right)$

$$
s=-\frac{2\left[S_{1} R_{2}+U_{1} \sqrt{R_{2}} \cos \xi\right]}{\left(S_{1}+\operatorname{ch} \varphi_{1}-1\right)\left(1-R_{2}\right)}
$$

где $R_{2}$ - коэффициент отражения просветляющей пленки,

$$
\begin{gathered}
\xi=2 \alpha+\sigma_{1}-\delta_{2}, \\
\alpha=k n h,
\end{gathered}
$$

$k$ - волновое число в вакууме, $n$ и $h$ - показатель преломления и толщина промежуточного слоя, $\delta_{2}$ - фаза амплитудного коэффициента отражения $r_{2}$ просветляющей пленки, т. е.

$$
r_{2}=\sqrt{R_{2}} e^{i \hat{\delta}_{2}}
$$

Далее, величины $S_{i}, \varphi_{1}$ и $U_{1} e^{i \sigma_{1}}$ характеризуют поглощающую пленку согласно формулам

$$
\begin{aligned}
S_{1} & =\frac{1}{2}\left(\frac{A_{1}}{D_{1}}-\frac{\tilde{A}_{1}}{\tilde{D}_{1}}\right) \\
\operatorname{ch} \varphi_{1} & =1+\frac{1}{2}\left(\frac{A_{1}}{D_{1}}+\frac{\tilde{A}_{1}}{\tilde{D}_{1}}\right) \\
U_{1} e^{i \sigma_{1}} & =\tilde{a}_{1} b_{1}-a_{1} \tilde{b}_{1}
\end{aligned}
$$

где означает, что величина характеризует сопряженную пленку (см. $[1,4,5])$, и 


$$
\left.\begin{array}{l}
a_{1}=\frac{r_{1}}{d_{1}}, \\
b_{1}=\frac{1}{d_{1}},
\end{array}\right\}
$$

а $r_{1}$ и $d_{1}$ - амплитудные коэффициенты отражения и пропускания просветляемой пленки. Отметим известное соотношение [3,4]

$$
U_{1}^{2}=S_{1}^{2}-\operatorname{sh}^{2} \varphi_{1}
$$

Входящие в формулу (2) величины $S_{1}, U_{1}, \varphi_{1}, R_{2}$ и $\xi$, от которых зависит $s$, все сами зависят от длины волны. Первые три из них зависят только от данной поглощающей пленки, а также (кроме $\varphi_{1}$ ) от показателя преломления промежуточного слоя. $R_{2}$ и $\xi$ зависят от просветляюией пленки и от показателей преломления промежуточного слоя и подложки, а $\xi$, кроме того, и от толщины этого слоя (через $2 \alpha$ ) и от просветляемой пленки (через $\sigma_{1}$ ). Таким образом, зависимость $s$ от длины волны довольно сложная. Поэтому целесообразно рассмотреть эту зависимость по частям.

\section{2. Зависимость $s$ от $R_{2}$ и $\xi$}

Пусть в формуле (2) величины $S_{1}, U_{1}$ и $\varphi_{1}$ имеют определенные значения, т. е. пусть поглошаюшая пленка и показатель преломления промежуточного слоя заданы, а длина волны фиксирована. Тогда формула (2) определяет для каждого значения $s$ некоторую зависимость между $\sqrt{R_{2}}$ и $\cos \xi:$

$$
\cos \xi=-\frac{S_{1}}{U_{1}} \sqrt{R_{2}}-\frac{\left(S_{1}+\operatorname{ch} \varphi_{1}-1\right) s}{2 U_{1}}\left(\frac{1}{\sqrt{R_{2}}}-\sqrt{R_{2}}\right) .
$$

Изображая эту зависимость графически, получим семейство кривых. На рис. $1-3$ показано это семейство для пленки некоторого конкретного состава (см. ниже, следующий раздел) при трех длинах волн.

Из графиков, а также из формулы (11) вытекают следующие характерные особенности рассматриваемой зависимости. Прямая линия $\cos \xi=-\frac{S_{1}}{U_{1}} \sqrt{R_{2}}$ соответствует значению $s=0$. Она делит плоскость $\left(\sqrt{R_{2}}, \cos \xi\right)$ в границах $0 \leqslant \sqrt{R_{2}} \leqslant 1,-1 \leqslant \cos \xi \leqslant 1$ на две неравные части. В меньшей части (внизу) $s>0$; кривые $s=$ const имеют здесь каждая по одному максимуму, причем все максимумы лежат на кривой

$$
\cos \xi=-\frac{2 S_{1}}{U_{1}\left(\sqrt{R_{2}}+\frac{1}{\sqrt{R_{2}}}\right)}
$$

В большей (верхней) части $s<0$, причем кривые $s=$ const экстремумов не имеют. Далее, все кривые $s=$ const $(s \gtrless 0)$ сходятся в точку $\sqrt{R_{2}}=1, \cos \xi=-\frac{S_{1}}{U_{1}}$, лежащую, однако, вне чертежа, так как всегда $\frac{S_{1}}{U_{1}}>1$ (см. (10)). Возможные значения $s$ снизу не ограничены, а сверху ограничены, причем 


$$
S_{\max }=\frac{S_{1}-\operatorname{sh} \varphi_{1}}{S_{1}+\operatorname{ch} \varphi_{1}-1} .
$$

Соответствующие значения $\cos \xi$ и $\sqrt{R_{2}}$ суть следующие:

$$
\left.\begin{array}{l}
\cos \xi=-1 \\
\sqrt{R_{2}}=\frac{S_{1}-\operatorname{sh} \varphi_{1}}{U_{1}}
\end{array}\right\}
$$

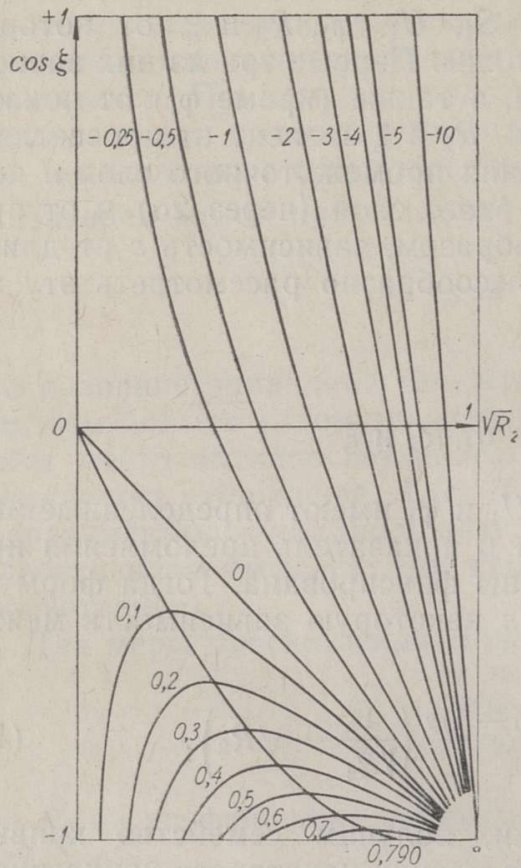

Рис. 1.

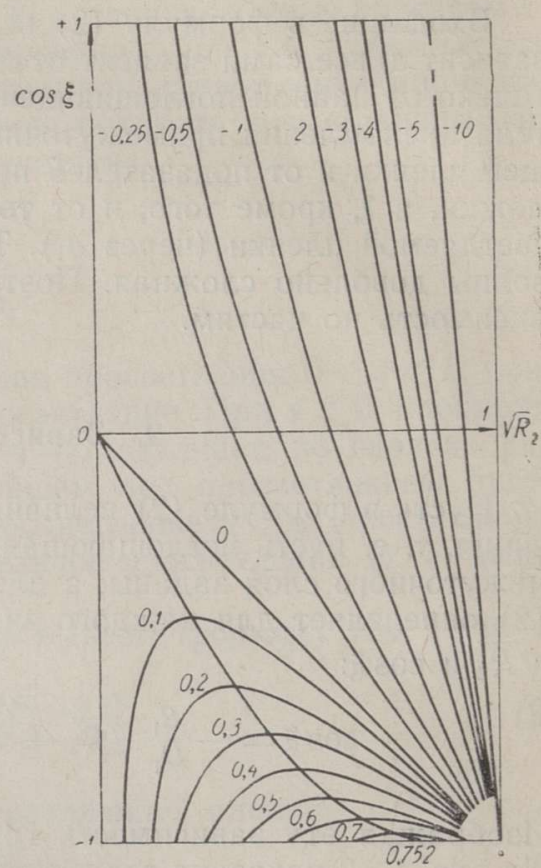

Рис. 2.

\section{3. Зависимость $s$ от длины волны при малых значениях $\varphi_{1}$}

Формула (11) определяет, в первую очередь, зависимость $s$ от $R_{2}$ и $\xi$, а через них и от длины волны. Но кроме того, $s$ зависит от длины волны также через $S_{1}, U_{1}$ и $\varphi_{1}$. Покажем, однако, что если величина $\varphi_{1}$ (которая, как известно, зависит только от просветляемой пленки) достаточно мала, то зависимость $s$ от длины волны через $S_{1}, U_{1}$ и $\varphi_{1}$ слаба - несмотря на то, что сами эти величины зависят от длины волны достаточно сильно. В самом деле, разлагая сh $\varphi_{1}$ и $U_{1}$ по степеням $\varphi_{1}$ и ограничиваясь членами степени не выше второй, находим:

$$
\begin{gathered}
\cos \xi=-\left(1-\frac{s}{2}\right) \sqrt{R_{2}}-\frac{s}{2 \sqrt{R_{2}}}- \\
-\frac{\varphi_{1}^{2}}{4 S_{1}^{2}}\left[2 \sqrt{R_{2}}+s\left(S_{1}+1\right)\left(\frac{1}{\sqrt{R_{2}}}-\sqrt{R_{2}}\right)\right]
\end{gathered}
$$


Чтобы оценить применимость этого приближения, заметим, что всегда имеет место неравенство (оно легко вытекает из (10), с учетом, что $\frac{A_{1}}{D_{1}}=\operatorname{ch} \varphi_{1}-1+S_{1}>0 ;$ см. также $\left.\left[{ }^{3}\right]\right)$

$$
\frac{\operatorname{ch} \varphi_{1}-1}{S_{1}}<1
$$

\section{Отсюда}

$$
\frac{\varphi_{1}^{2}}{2 S_{1}}<1
$$

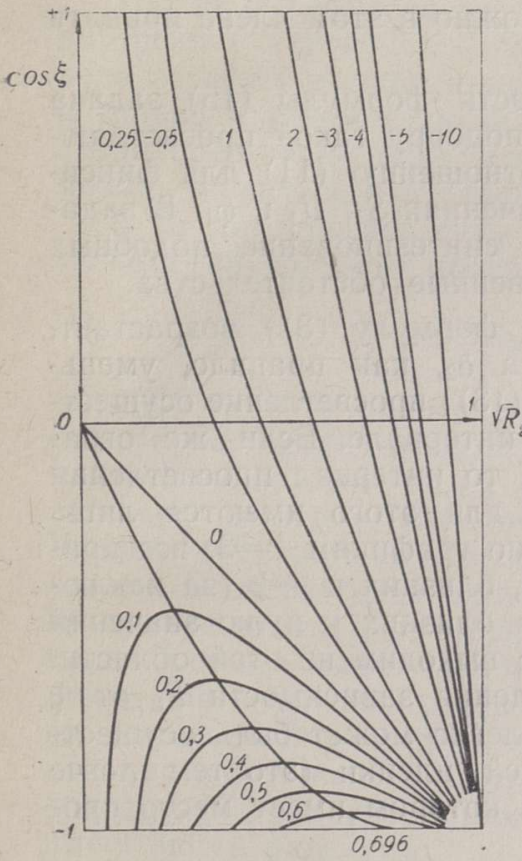

Рис. 3

Кроме того, из той же формулы (10) следует, что

$$
\frac{\varphi_{1}^{2}}{S_{1}^{2}}<1 .
$$

Легко видеть, что неучтенные в (15) члены разложения, содержащие $\varphi_{1}^{2}, \varphi_{1}^{4}$ и т. д., убывают в силу (17) и (18) достаточно быстро, особенно если $\varphi_{1}^{2} \ll 1$ и даже если $\varphi_{1}^{2} \lesssim 1$. Мал также, вообще говоря, и последний член в формуле (15) (по сравнению с двумя первыми членами правой части). Этот член тем меньше, чем лучше выполняются неравенства (17) и (18) и чем меньше $\varphi_{1}^{2}$.

Но так как первые два члена в правой части формулы (15) не зависят от $S_{1}, U_{1}$ и $\varphi_{1}$, то это и означает, что зависимость $s$ от длины волны осуществляется главным образом только через $R_{2}$ и $\xi$.

В частности, неравенства (17) и (18) всегда хорошо выполняются для одиночного металлического (или, вообще, поглощающего) слоя, если он только не настолько толст, что практически непрозрачен. Кроме того, для такого, не слишком толстого поглощающего слоя всегда $\varphi_{1}^{2} \ll 1$. Следовательно, приближенная формула (15) в этом случае хорошо применима. В качестве примера на рис. $1-3$ изображены семейства кривых $s=$ const при трех длинах волн $(6300 \AA ̊ 25400 \AA ̊$ и $4500 \AA$ ) для слоя серебра толщиной $200 \AA ̊$. Показатель преломления $\eta_{1}-i x_{1}$ серебра принят равным при этих длинах волн, соответственно, 0,24 $-4,34 i, 0,19-3,60 i, 0,17-2,71 i$. Эти значения можно считать приближенно верными, как вытекает из экспериментальных данных статьи [6]*. Показатель преломления промежуточного слоя принят равным $n=2,35$ $(\mathrm{ZnS})$, так как, по некоторым соображениям (см. [1]), целесообразно, чтобы он был возможно большим. В нижеследующей таблице приве дены значения $S_{1}, U_{1}, \varphi_{1}$ и $\sigma_{1}$ для такого слоя (дисперсия $n$ не учитывается).

* Отметим, что в статье $\left[{ }^{7}\right]$ показатель преломления серебра принят ошибочно равным $0,27-2,5 i$; эта ошибка не влияет, однако, на принципиальные результаты указанной статьн. 


\begin{tabular}{c|c|c|c|c}
\hline$\lambda$ & $S_{1}$ & $U_{1}$ & $\phi_{1}$ & $\sigma_{1}$ \\
\hline $6300 \AA$ & 0,248 & 0,243 & 0,051 & $26^{\circ}$ \\
$5400 \AA$ & 0,190 & 0,184 & 0,046 & $31^{\circ}$ \\
$4500 \AA$ & 0,149 & 0,142 & 0,044 & $37^{\circ}$
\end{tabular}

Как видим из графиков, действительно, при всех длинах волн общая картина сходна. Близкие значения имеют и $s_{\max }$ (см. формулу (13)), именно, 0,790, 0,752 и 0,696. Следовательно, в первом приближении, изучая зависимость $s$ от длины волны, можно пренебречь зависимостью $S_{1}, U_{1}$ и $\varphi_{1}$ от длины волны. Это не значит, что в формуле (15) можно отбросить последний член, но для $\varphi_{1}$ и $S_{1}$ можно в этом члене принять некоторые средние значения.

Таким образом, при условии применимости формулы (15) задача об ахроматичном просветлении сводится к подбору такой просветляющей пленки, которая удовлетворяла бы соотношению (11) для фиксированного $s$ при некоторых усредненных значениях $S_{1}, U_{1}$ и $\varphi_{1}$. В задачу настоящей статьи не входит подробное синтезирование подобных пленок. Отметим, однако, наиболее существенные обстоятельства.

1. С уменьшением длины волны $\xi$ (см. формулу (3)) возрастает, так как $\alpha$ и $\sigma_{1}$ (см. табл.) возрастают, а $\delta_{2}$, как правило, уменьшается. Поэтому максимальное (по формуле (13)) просветление осуществимо только в весьма узком спектральном интервале. Если же ограничиться худшим просветлением $\left(s<s_{\max }\right)$, то интервал просветления может быть несколько расширен. Однако и для этого имеются лишь ограниченные возможности, так как, согласно графикам $1-3$, все кривые $c s>0$ лежат в области значений $\cos \xi$, близких $\mathrm{k}-1$ (за исключением только не представляющих интереса близких к нулю значений $s)$, так что с возрастанием $\xi$ мы неизбежно выходим из этой области. Что же касается необходимой для просветления зависимости $R_{2}$ от $\xi$ (по формуле (11)), то она, вообще говоря, легко может быть осуществлена надлежащим подбором просветляющей пленки (это тем легче сделать, чем у́же спектральный интервал, в котором имеет место просветление).

2. Иначе обстоит дело с антипросветлением. Возрастание $\xi$ с уменьшением длины волны не препятствует сохранению антипросветления, так как кривые с $s<0$ простираются на все значения $\cos \xi$ от -1 до +1. Для осуществления ахроматического антипросветления в широком спектральном интервале следует лишь позаботиться о надлежащей зависимости между $R_{2}$ и $\xi$. Принципиально это возможно сделать, и притом, вообще говоря, нетрудно, но, конечно, тем труднее, чем шире интервал спектра и чем больше $|s|$.

Важно, наконец, подчеркнуть следующее. В первом из этих двух пунктов говорится о возрастании $\sigma_{1}$ с уменьшением длины волны. Это, действительно, имеет место всегда для единичного поглощающего слоя. Может возникнуть вопрос, так ли это для пленки, содержащей более одного поглощающего слоя. Қак увидим в следующем разделе, учет внутреннего просветления заставляет ответить на этот вопрос утвердительно. 


\section{4. Учет внутреннего просветления или антипросветления}

В предыдущем разделе мы рассмотрели зависимость $S$ от длины волны и вытекающие отсюда условия ахроматического просветления или антипросветления при условии достаточной малости $\varphi_{1}$, что, в частности, выполняется для отдельного металлического слоя. Если же пленка содержит несколько поглощающих слоев, то $\varphi_{1}$ может иметь большие значения. Все формулы (2)-(14) в этом случае остаются в силе; мы можем также всегда построить диаграмму, подобную приведенным на рис. 1 -3. Однако формула (15) становится уже неприменимой (хотя неравенства (17) и (18) верны всегда). Поэтому зависимость $s$ от длины волны становится более сложной.

Тем не менее, если мы, наряду со внешним, будем учитывать также внутреннее (анти)просветление, то главный вывод предыдущего раздела остается в силе: просветление поддается ахроматизации с трудом и только в узком спектральном интервале, тогда как ахроматическое антипросветление возможно в широкой спектральной области. В этом можно убедиться следующим образом.

В случае просветления в собственном смысле этого слова одно из условий внутреннего просветления гласит:

$$
\cos \left(2 \alpha_{k}+\sigma_{k-1, k}+\sigma_{k, k+1}^{\prime}\right)=1
$$

(см. $\left.\left.{ }^{2}\right]\right)$, где $k$ - индекс какого-либо промежуточного слоя, находящегося между двумя соседними слоями остова с индексами $k-1, k$ и $k, k+1 . \alpha_{k}, \sigma_{k-1}, k$ и $\sigma_{k, k+1}^{\prime}$ определяются подобно $\alpha$ и $\sigma_{1}$ в формулах (4) и (8), а штрих' означает, что свет падает в обратном направлении. Условие (19) должно выполняться для всех промежутков между слоями астова. Если вдобавок выполняется еще другое условие (его мы здесь не приводим, см. $\left.\left[{ }^{2}\right]\right)$, то пленка просветлена внутренне, и тогда $\varphi_{1}$ имеет для нее минимальное возможное значение, равное сумме значений ф для отдельных слоев остова. Будем считать, что эта сумма меньше единицы. Это предположение является разумным и реальным, так как иначе пленка была бы практически непрозрачна. Кроме того, как показано [2], в случае выполнения условий внутреннего просветления, имеет место равенство

$$
\sigma_{N-1, N}=\sigma_{1}
$$

$(N-$ число слоев остова), т. е. фигурирующая в формуле (3) величина $\sigma_{1}$, характеризуюшая всю просветляемую пленку в целом, равна аналогичной величине, относящейся к последнему слою остова. Это значит, что $\sigma_{1}$, как и в случае единичного поглощающего слоя, возрастает с уменьшением длины волны. Следовательно, при наличии внутреннего просветления оказывается полностью применимой для внешнего просветления та теория, которую мы развили в предыдущих разделах, вместе с ее основным выводом о невозможности ахроматизации просветления в широкой области. Фактически возможности ахроматизации сужаются теперь еще более, поскольку выполнение условия (19), а также другого условия внутреннего просветления тоже зависит от длины волны. Но даже в случае, если эти условия можно сделать ахроматичными полностью, невозможность широкой ахроматизации просветления остается в силе.

Иначе обстоит дело в случае антипросветления. Для того, чтобы сделать $A / D$ возможно большим, нужно иметь возможно большое значение $A_{1} / D_{1}$ и возможно большое по абсолютной величине отрицатель- 
ное значение $s$. Первое условие может быть выполнено надлежащим выбором прослоек между слоями остова, причем если даже действие этих прослоек на значение $A_{1} / D_{1}$ не обладает достаточной ахроматичностью, все же можно, применяя внешнее антипросветление, добиться такой зависимости $S$ от длины волны, при которой $A / D$ будет сохранять более или менее постоянное значение в широком спектральном интервале. Это вытекает из характера зависимости $s$ от $\overline{V R}_{2}$ и $\cos \xi$, как показано в предыдущих разделах. В детали конкретного осуществления подобной возможности мы здесь не входим, так как цель настоящей статьи состоит только в установлении общих положений о возможностях ахроматизации просветления или антипросветления.

\title{
ЛИТЕ РА Т У Р А
}

1. П. Г. Кард, Теория просветления металлических покрытий. Оптика и спектроскопия, 9, 2, 1960.

2. П. Г. К а рд, Основы теории синтеза просветленных поглощающих покрытий. Оптика и спектроскопия, 9, 3, 1960.

3. П. Г. К а рд, К теории поглощающих оптических покрытий. Изв. АН ЭССР. Сер. физ.-матем. и техн. наук, 9, 3, 1960.

4. P. K a rd, Uusi tulemusi neelavate optiliste katete teoorias. Loodus ja matemaatika, nr. 4, в печати.

5. П. Г. К а рд, Новые рекуррентные формулы в теории многослойных оптических покрытий. Оптика и спектроскопия, 9, 1, 1960.

6. R. C. F a ust, An interferometric study of some optical properties of evaporated silver films. Phil. Mag., 41, 323, 1950.

7. П. Г. К а рд, Теория просветленного светофильтра типа Фабри-Перо. Изв. АН ЭССР. Сер. физ.-матем. и техн. наук, 11, 3, 1962.

Тартуский государственный университет

Поступила в редакцию

25. V 1962

\section{NEELAVATE KELMETE SELGENDUSE VÕ ANTISELGENDUSE AKROMATISM}

\author{
P. Kard, \\ Eesti NSV Teaduste Akadeemia korrespondeeriv liige \\ Resümee
}

Neelava kelme selgenduse mōõdu $s$ üldise valemi (2) alusel analüüsitakse selgenduse või antiselgenduse akromaatsuse tingimusi. Näidatakse, et selgenduse akromatism on saavutatav ainult võrdlemisi kitsas spektrivahemikus, kuna antiselgendust saab lainepikkusest nõrgalt olenevaks teha laias spektripiirkonnas.

Tartu Riiklik Olikool

Saabus toimetusse

25. V 1962

\section{ACHROMATISM OF CLARIFICATION OR ANTICLARIFICATION OF ABSORBING FILMS}

\author{
P. Kard, \\ Corresponding Member of the Academy of Sciences \\ of the Estonian S.S.R.
}

Summary

On the basis of the general formula (2) for the measure $s$ of the clarification of an absorbing film an analysis of the conditions of achromatism of clarification or anticlarification is given. It is shown that achromatism of clarification is attainable only in a relatively narrow spectral interval, whereas anticlarification can be made slightly depending on the wavelength in a broad spectral region. 